\title{
An unusual complication during implantation of an internal cardioverter-defibrillator: A Case Report
}

Shveta Monga ${ }^{1}$ MBBS MRCP, Atique Imam² MB BS FRCS FRCR, Richard Bulbulbia ${ }^{3}$ MA MD FRCS, Paul Foley ${ }^{1}$ MD FRCP, William Mc Crea ${ }^{1}$ MB BCh FRCP, Badrinathan Chandrasekaran ${ }^{1}$ BSC MD(Res) FRCP

Monga S, Imam A, Bulbulbia R, et al. An unusual complication during implantation of an internal cardioverter-defibrillator: A Case Report. Curr Res Cardiol 2017;4(2):14.

Subclavian arteriovenous fistulas are a rare but known complication of catheterisation procedures, pacemaker and internal cardioverter-defibrillator (ICD) implantation and surgery. These fistulas typically become apparent few days after procedure through clinical signs of a high output circulation. However, small fistulas can remain asymptomatic. We describe a case of a chronic Subclavian Arteriovenous Fistula (AVF) complicating an otherwise straightforward ICD device implantation procedure. The patient required urgent endovascular treatment to close the fistula.

Key Words: Mitral valve replacement; Rheumatic heart disease; Angiography; Arteriovenous fistula
A 62-year-old Caucasian male patient underwent a single chamber AICD insertion for primary prevention. He had been admitted with a spontaneous left rectus sheath haematoma and his admission was complicated by a hospital acquired pneumonia and non-sustained monomorphic VT. His past medical history included mechanical aortic and mitral valve replacement for rheumatic heart disease in 1996, type 2 diabetes mellitus, permanent atrial fibrillation and incomplete L1 paraplegia. Echocardiography demonstrated severe left ventricular dysfunction with an ejection fraction of less than $30 \%$. Pre-procedure, there were no physical signs of congestion with equal upper limb pulses bilaterally and no prominence of veins over the upper chest wall. There was no palpable thrill or audible bruit on examination. His warfarin dose had been adjusted and his INR on the day of the procedure was 1.6.

A delto-pectoral groove incision was made and access obtained blindly, without a venogram, via the left subclavian vein using the extra thoracic approach with no complication. The left cephalic vein was atretic and not able to be used. An active 8 French single coil DF 4 active lead was placed in RV apex using a 9 French safe sheath. Persistent pulsatile arterial bleeding was noticed on removal of the safe sheath at the subclavian vein puncture site with no clear visible source. Simple measures, including hand held pressure and use of purse string sutures, were unable to stem the bleeding. Active RV lead was left insitu and the vascular surgical team were consulted. Selective angiography of the aortic arch and subclavian artery revealed a chronic fistula between left subclavian artery and left subclavian vein proximal to the insertion of the defibrillator lead (Figure 1A). There was no extravasation in the surrounding tissue to suggest an acute fistula caused by an inadvertent arterial puncture. Also, selective angiography (Figure 1A) showed clearly that the defibrillator lead inserted directly into the left subclavian vein and did not pass through the fistula further supporting the chronic nature of the fistula $(1-3)$.
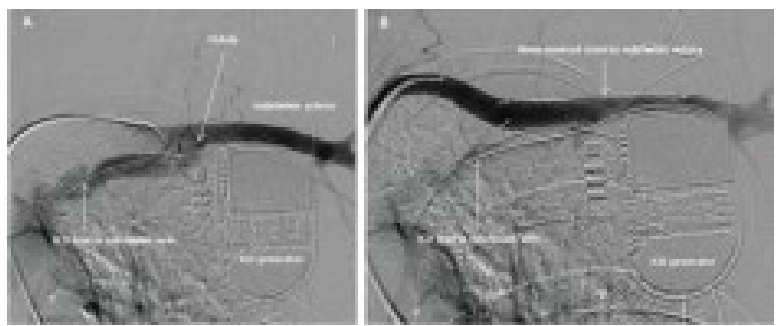

Figure 1) Arch aortogram with catheter in the left subclavian artery demonstrating a fistula to the subclavian vein proximal to the internal defibrillator (ICD) lead insertion (panel A). Following the stent there is no longer any contrast passing from artery to vein (panel B).
A $9 \mathrm{~mm}$ covered stent was placed in the subclavian artery which stemmed the bleeding and allowed closure of the wound (Figure 1B). Unfractionated heparin was started the following day and he was re-established on a therapeutic dose of warfarin. Patient has since been followed up in the outpatient clinic and continues to do well.

\section{DISCUSSION}

This case illustrates an unusual presentation of a chronic arteriovenous fistula which was not clinically suspected at the time of a routine ICD insertion procedure. There was no mention of any such anomalous finding in patient's previous operation notes. We suspect that the fistula may have been caused by central line insertion at the time of his original surgery.

Subclavian vein catheterisation is seldom complicated by AVF formation. Incidence of this complication is estimated to be less than $0.58 \%$ (1). There are a few case reports of iatrogenic arteriovenous fistula following median sternotomy (2) but these are usually apparent clinically soon after, hence that seems unlikely in our case. An alternative explanation is a congenital fistula (3) but the lack of clinical signs makes this less probable. To our knowledge, this is the first described case of a chronic AVF complicating pacing/ICD lead insertion in the literature and we demonstrate a novel minimally invasive solution to achieve procedural success.

\section{REFERENCES}

1. Chloroyiannis Y, Reul GJ. Iatrogenic left subclavian artery-to-left brachiocephalic vein fistula. Tex Heart Inst J 2004;31:172-74.

2. Thiel R, Bircks W. Arteriovenous fistulas after median sternotomyreport of 2 cases and review of the literature. Thorac Cardiovasc Surg 1990;38:195-97.

3. Konstantin S, David WP. Congenital subclavian arteriovenous fistula. BMJ Case Rep 2009.

${ }^{1}$ Wiltshire Cardiac Centre, Great Western Hospital, Swindon SN3 6BB, UK, ${ }^{2}$ Department of Interventional Radiology, Great Western Hospital, Swindon SN3 6BB, UK, ${ }^{3}$ Department of Vascular Surgery, Cheltenham General hospital, Cheltenham GL53 7AN, UK

Correspondence:Shveta Monga MBBS MRCP, Wiltshire Cardiac Centre, Great Western hospital, Swindon SN3 6BB, UK, Telephone 00447900422718, E-mail shveta. monga@gmail.com, shveta.monga@nhs.net

Received: February 13, 2017, Accepted: July 06, 2017, Published: July 08, 2017 